\title{
A microvariability study of nearby $M$ dwarfs from the Western Italian Alps: Status update
}

\author{
Mario Damasso ${ }^{1,2}$, Andrea Bernagozzi ${ }^{1}$, Enzo Bertolini ${ }^{1}$, Paolo \\ Calcidese $^{1}$, Paolo Giacobbe ${ }^{3}$, Mario G. Lattanzi ${ }^{4}$, Matteo Perdoncin ${ }^{5}$, \\ Alessandro Sozzetti ${ }^{4}$, Richard Smart $^{4}$ and Giorgio Toso ${ }^{1}$ \\ ${ }^{1}$ Astronomical Observatory of the Autonomous Region of the Aosta Valley, Loc. Lignan 39, \\ 11020 Nus (Aosta), Italy \\ ${ }^{2}$ Dept. of Astronomy, University of Padova, Vicolo dell'Osservatorio 5, I-35122 Padova, Italy \\ email: m.damasso@gmail.com \\ ${ }^{3}$ Dept. of Physics, University of Trieste, Via Tiepolo 11, I-34143 Trieste, Italy \\ ${ }^{4}$ INAF - Astronomical Observatory of Torino, Via Osservatorio 20, I-10025 Pino Torinese, Italy \\ ${ }^{5}$ Dept. of Physics, University of Torino, Via Giuria 1, I-10125 Torino, Italy
}

\begin{abstract}
Small ground-based telescopes can effectively be used to look for transiting rocky planets around nearby low-mass M stars, as recently demonstrated for example by the MEarth project. Since December 2009 at the Astronomical Observatory of the Autonomous Region of Aosta Valley (OAVdA) we are monitoring photometrically a sample of red dwarfs with accurate parallax measurements. The primary goal of this 'pilot study' is the characterization of the photometric microvariability of each target over a typical period of approximately 2 months. This is the preparatory step to long-term survey with an array of identical small telescopes, with kick-off in early 2011. Here we discuss the present status of the study, describing the stellar sample, and presenting the most interesting results obtained so far, including the aggressive data analysis devoted to the characterization of the variability properties of the sample and the search for transit-like signals.
\end{abstract}

Keywords. techniques: photometric, planetary systems

\section{Introduction}

Since December 2009 a photometric survey of a small sample of nearby M dwarfs has been carrying out at the Astronomical Observatory of the Autonomous Region of the Aosta Valley (OAVdA), using less than 1-meter class telescopes. The aim of this 1-year long observing campaign is the monitoring of the microvariability of the stars over a period of some weeks to assess the implications for the detection of small-size transiting planets. The study represents a preparatory step toward a long-term survey of thousands of red dwarfs aimed at the detection of rocky planets. The OAVdA has been chosen as the hosting institution for the campaign on the base of the results of a site characterization study (Damasso et al. 2010).

\section{Instrumentation and methodology}

The instrumental set up used in this study is detailed in Damasso et al. (2010) and is composed of a 810, 400 and $250 \mathrm{~mm}$ telescope. We selected the targets from a list of nearby M dwarfs with precise parallaxes observed by Smart et al. (2010). On average each target has been monitored for $3 \mathrm{hr} /$ night for a maximum period of 2 months. In parallel to the observations the data reduction and analysis pipeline TEEPEE (Transiting 


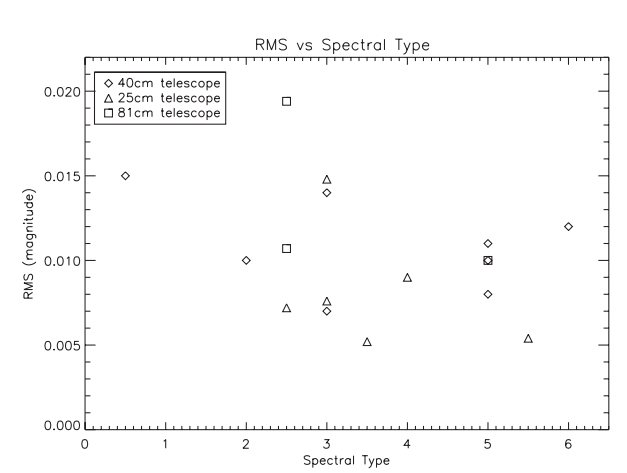

Figure 1. RMS of the target light curves as a function of target spectral subtype (M0-M6).

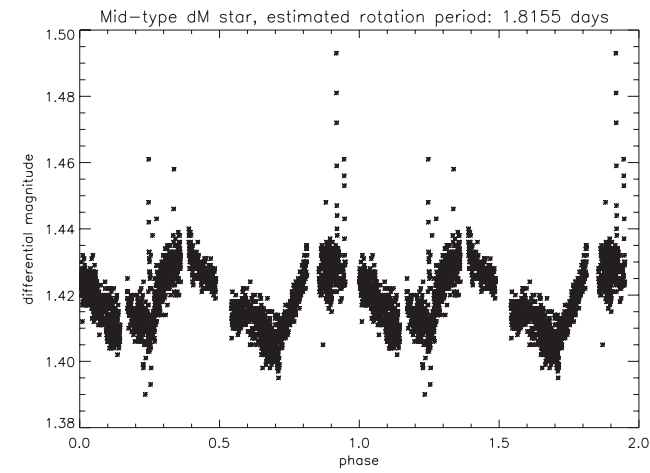

Figure 2. Differential light curve of a $\mathrm{M}$ dwarf folded according to the tentative rotation period of 1.8155 days.

ExoplanEts PipElinE; Damasso et al. 2010) has been upgraded, e.g. now including the SysRem algorithm to correct systematic effects in the light curves (Tamuz et al. 2005).

\section{Some preliminary results}

Fig. 1 shows the RMS of the light curves of some targets of our sample organized according to their spectral subtype. Each RMS is calculated for the whole period of observation. No correlation is evident from the data. Fig. 2 shows the differential light curve of a mid-type $\mathrm{M}$ dwarf which is made up of 19 observing sessions and is folded according the tentative rotation period of 1.8155 days, under the hypothesis that the observed modulations depend on the visibility conditions of active regions or star spots on the stellar disk. Three stellar flares are visible in the data, which were observed in two almost consecutive nights.

\section{Toward a long-term automated survey}

With kick off in Spring 2011, a long-term automated survey of thousands of nearby M dwarfs will start at the OAVdA, using an array of dedicated $400 \mathrm{~mm}$ telescopes. The main aim of the campaign will be the detection of small-size transiting extrasolar planets, designing an Europe-based observational program similar to the US MEarth project which has already discovered the super-Earth GJ 1214b (Charbonneau et al. 2009).

\section{Acknowledgements}

MD, PC, AB, and GT are supported by grants of the European Union, the Autonomous Region of the Aosta Valley and the Italian Department for Work, Health and Pensions. The OAVdA is supported by the Regional Government of Valle d'Aosta, the Town Municipality of Nus and the Monte Emilius Community.

\section{References}

Charbonneau, D., Berta, Z. K., Irwin, J., et al. 2009, Nature, 462, 891

Damasso, M., Giacobbe, P., Calcidese, P., et al. 2010, PASP, 122, 895, 1077

Smart, R. L., Ioannidis, G., Jones, H. R. A., et al. 2010, A\&SA, 514, A84

Tamuz, O., Mazeh, T., \& Zucker, S. 2005, MNRAS, 356, 4, 1466 